\title{
Energy loss of heavy quarkonia in hot QCD plasmas
}

\author{
Juhee Hong $\odot$ and Su Houng Lee $\odot$ \\ Department of Physics and Institute of Physics and Applied Physics, Yonsei University, Seoul 03722, Korea
}

(Received 4 March 2021; accepted 3 May 2021; published 18 May 2021)

\begin{abstract}
We compute the energy loss of heavy quarkonia in high-temperature QCD plasmas and investigate the energyloss effects on quarkonium suppression. Based on the effective vertex derived from the Bethe-Salpeter amplitude for quarkonium, the collisional and radiative energy loss are determined by quarkonium-gluon elastic scattering and the associated gluon-bremsstrahlung, respectively. In the energy regime $E<m_{\Upsilon}^{2} / T$ the collisional energy loss is dominant over the radiative one, and the total energy loss increases with the plasma temperature and the initial energy of quarkonium. Our numerical analysis indicates that the medium-induced energy loss of the $\Upsilon(1 S)$ results in stronger suppression at higher momentum, although the energy-loss effects are found to be small compared with the previous estimates of quarkonium dissociation in heavy-ion collisions.
\end{abstract}

DOI: 10.1103/PhysRevC.103.054907

\section{INTRODUCTION}

The depletion of high-momentum particle production with respect to $p p$ collisions signals the formation of a quark-gluon plasma (QGP) in heavy-ion collisions. Especially, heavy quarks and quarkonia which are mostly formed from the initial fusion of partons at an early stage are important probes to investigate the transport and thermal properties of the hightemperature and -density matter. While quarkonia suppression can be influenced by various mechanisms including dissociation and energy loss, the energy loss of heavy quarkonia has not been computed and is not described by the heavy quark-antiquark potential.

Energetic particles traversing QCD plasmas suffer energy loss by elastic scattering or gluon-bremsstrahlung: drag and diffusion cause particles to lose their energies, and incoming high-energy particles can be radiatively deprived of fractions of their energies. There have been many studies about the energy loss of partons. The diffusion processes are dominated by $t$-channel gluon exchange with soft momentum transfer which requires hard-thermal-loop resummation $[1,2]$. In the presence of multiple scatterings, destructive interference occurs when the formation time is large compared with the mean-free path [3-6]. The radiative energy loss is naively of order $g^{2}$ higher than the collisional one, but there can be enhancement in the limit of soft and collinear emission: both radiative and collisional processes of heavy quarks can be of the same order in the coupling constant, $-\frac{d E}{d x} \sim g^{4} T^{2}$ neglecting logarithmic corrections [2,7]. The radiative energy loss is dominant over the collisional one for ultrarelativistic partons, whereas the collisional energy loss is not negligible for heavy quarks [8-10].

Published by the American Physical Society under the terms of the Creative Commons Attribution 4.0 International license. Further distribution of this work must maintain attribution to the author(s) and the published article's title, journal citation, and DOI.
We are interested in the energy loss of a color singlet quarkonium in QCD media such as QGPs or large nuclei. After production at an early stage of heavy-ion collisions, quarkonia undergo not only dissociation (and regeneration) but also energy loss. A quarkonium state loses its energy by elastic scattering $(g+\Upsilon \rightarrow g+\Upsilon)$ which can induce gluon radiation. Quarkonium diffusion and energy loss have been discussed with potential nonrelativistic QCD (pNRQCD) in the regime $E_{b} \gtrsim T$ [11]. In this work, we will use a formalism developed in our previous work [12]: for quarkonium dissociation through the color-dipole interaction [13], an effective vertex based on the Bethe-Salpeter amplitude has been introduced to calculate the next-to-leading order cross sections which agree with the results of pNRQCD in the regime $T \gtrsim$ $E_{b}$ [14]. In the current kinematic range up to $q_{T} \sim 30 \mathrm{GeV}$ [15], heavy quarkonia are not ultrarelativistic $\left(E<m_{\Upsilon}^{2} / T\right)$ and thus the collisional energy loss can be considerable.

We will discuss how the energy loss of heavy quarkonia can be calculated using our formalism of the effective vertex, and estimate the energy-loss effects on quarkonium spectrum in heavy-ion collisions. In Sec. II, we calculate the momentum diffusion coefficient and the collisional energy loss of weakly bound quarkonia at high temperature. In Sec. III, we discuss the radiative energy loss by gluon-bremsstrahlung associated with quarkonium-gluon elastic scattering. Shifting the transverse momentum spectra of the $\Upsilon(1 S)$ by its mean energy loss, we estimate the energy-loss effects on the nuclear modification factor in Sec. IV. Finally, a summary is given in Sec. V.

\section{COLLISIONAL ENERGY LOSS}

We are interested in diffusion and energy loss of weakly bound quarkonia in a high-temperature regime where the binding energy is smaller than the temperature scale. Due to the high melting temperature $T_{\text {melt }} \approx 600 \mathrm{MeV}$ [16], the ground state of bottomonium survives as a color singlet and undergoes diffusion in QGP below $T_{\text {melt }}$. 


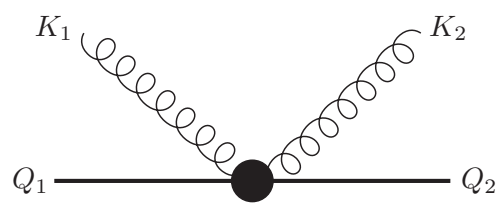

(a)

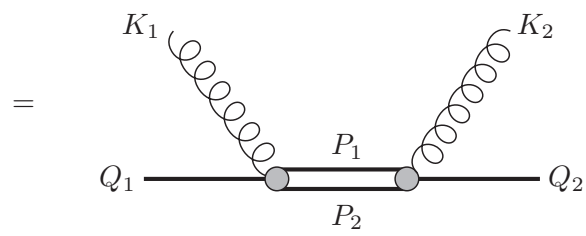

(b)

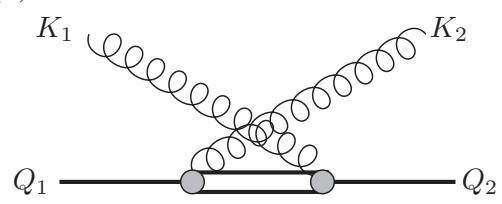

FIG. 1. Elastic scattering $(g+\Upsilon \rightarrow g+\Upsilon)$ contributing to quarkonium diffusion and energy loss.

To determine the momentum diffusion coefficient of heavy quarkonia, we consider quarkonium-gluon elastic scattering. In Figs. 1(a) and 1(b), the dipole interaction of color charge with gluon can be described by the following effective vertex derived from the Bethe-Salpeter amplitude [17]:

$$
\begin{aligned}
V^{\mu \nu}(K)= & -g \sqrt{\frac{m_{\Upsilon}}{N_{c}}}\left[\boldsymbol{k} \cdot \frac{\partial \psi(\boldsymbol{p})}{\partial \boldsymbol{p}} \delta^{\mu 0}+k_{0} \frac{\partial \psi(\boldsymbol{p})}{\partial p^{i}} \delta^{\mu i}\right] \\
& \times \delta^{\nu j} \frac{1+\gamma^{0}}{2} \gamma^{j} \frac{1-\gamma^{0}}{2} T^{a},
\end{aligned}
$$

where $\boldsymbol{p}=\left(\boldsymbol{p}_{1}-\boldsymbol{p}_{2}\right) / 2$ is the relative momentum between heavy quark and antiquark. With two effective vertices and heavy quark propagators $\Delta(P)$, the quarkonium-gluon elastic scattering in Fig. 1(a) has the following amplitude [11]:

$$
\begin{aligned}
\mathcal{M}_{\mathrm{el}}^{\mu \nu \rho \sigma(a)}= & \int \frac{d^{4} P}{(2 \pi)^{4}} \Delta\left(P_{1}\right) V^{\mu \nu}\left(K_{1}\right) \Delta\left(P_{2}\right) V^{\rho \sigma *}\left(K_{2}\right) \\
= & i \frac{g^{2} m_{\Upsilon}}{2 N_{c}} \delta^{a b} k_{10} k_{20} \int \frac{d^{3} \boldsymbol{p}}{(2 \pi)^{3}} \frac{\partial \psi(\boldsymbol{p})}{\partial p^{i}} \delta^{\mu i} \frac{\partial \psi(\boldsymbol{p})}{\partial p^{k}} \delta^{\rho k} \\
& \times \frac{\delta^{\nu j} \delta^{\sigma l} \operatorname{Tr}\left[\gamma^{j} \gamma^{l}\right]}{2\left(k_{10}-E_{b}-\frac{\boldsymbol{p}^{2}}{m}\right)},
\end{aligned}
$$

and similarly for Fig. 1(b) except the denominator has $2\left(-k_{20}-E_{b}-\frac{p^{2}}{m}\right)$. For $k_{10}, k_{20} \gg E_{b}$, the total matrix element squared (averaged over the quarkonium polarization) is

$$
\begin{aligned}
\left|\mathcal{M}_{\mathrm{el}}\right|^{2}= & \frac{32}{81} g^{4} m_{\Upsilon}^{2}\left(1+\cos ^{2} \theta_{k_{1} k_{2}}\right) \\
& \times\left[\int \frac{d^{3} \boldsymbol{p}}{(2 \pi)^{3}}\left(\frac{\boldsymbol{p}^{2}}{m}+E_{b}\right)|\nabla \psi|^{2}\right]^{2},
\end{aligned}
$$

where $\theta_{k_{1} k_{2}}$ is the angle between $\boldsymbol{k}_{1}$ and $\boldsymbol{k}_{2}$.

The momentum diffusion coefficient is defined by the mean-squared momentum transfer per unit time [8],

$$
\begin{aligned}
3 \kappa= & \frac{1}{2 q_{10}} \int \frac{d^{3} \boldsymbol{q}_{2}}{(2 \pi)^{3} 2 q_{20}} \int \frac{d^{3} \boldsymbol{k}_{1}}{(2 \pi)^{3} 2 k_{10}} \int \frac{d^{3} \boldsymbol{k}_{2}}{(2 \pi)^{3} 2 k_{20}} \\
& \times n\left(k_{1}\right)\left[1+n\left(k_{2}\right)\right](2 \pi)^{4} \delta^{4}\left(K_{1}+Q_{1}\right. \\
& \left.-K_{2}-Q_{2}\right)\left|\mathcal{M}_{\mathrm{el}}\right|^{2}\left(\boldsymbol{k}_{1}-\boldsymbol{k}_{2}\right)^{2}
\end{aligned}
$$

where $n(k)=1 /\left(e^{k / T}-1\right)$ is a thermal distribution of gluon. Using a Coulombic bound state, $\left|\nabla \psi_{1 S}(\boldsymbol{p})\right|^{2}=$ $2^{10} \pi a_{0}^{7} \boldsymbol{p}^{2} /\left[\left(a_{0} \boldsymbol{p}\right)^{2}+1\right]^{6}$, with $a_{0}^{2}=1 /\left(m E_{b}\right)$ which is satisfied for the Coulombic binding energy, we have

$$
\kappa=\frac{128 \pi g^{4} T^{5}}{1215 m^{2}} .
$$

The diffusion coefficient of quarkonium is suppressed by $T^{2} / m^{2}$ compared with the heavy quark diffusion $\kappa_{\mathrm{HQ}} \sim g^{4} T^{3}$ [8]. In the QGP temperature region, Eq. (5) is of the same order of magnitude as the results in Ref. [11] but is much smaller than the momentum broadening rate for tightly bound quarkonia in Ref. [18].

In the energy regime $E<m_{\Upsilon}^{2} / T$, the collisional energy loss per unit length is obtained by the interaction rate of quarkonium weighted by $\left(k_{2}-k_{1}\right) / v(v$ is the quarkonium velocity) $[1,2]$

$$
\begin{aligned}
-\frac{d E}{d x}= & \frac{1}{2 q_{10}} \int \frac{d^{3} \boldsymbol{q}_{2}}{(2 \pi)^{3} 2 q_{20}} \int \frac{d^{3} \boldsymbol{k}_{1}}{(2 \pi)^{3} 2 k_{10}} \\
& \times \int \frac{d^{3} \boldsymbol{k}_{2}}{(2 \pi)^{3} 2 k_{20}} n\left(k_{1}\right)\left[1+n\left(k_{2}\right)\right](2 \pi)^{4} \\
& \times \delta^{4}\left(K_{1}+Q_{1}-K_{2}-Q_{2}\right)\left|\mathcal{M}_{\mathrm{el}}\right|^{2} \frac{\left(k_{2}-k_{1}\right)}{v},
\end{aligned}
$$

where $\cos \theta_{k_{1} k_{2}}$ in Eq. (3) is rewritten in a covariant form,

$$
\cos \theta_{k_{1} k_{2}}=1-\frac{\left(K_{1} \cdot K_{2}\right) m_{\Upsilon}^{2}}{\left(K_{1} \cdot Q_{1}\right)\left(K_{2} \cdot Q_{1}\right)} .
$$

The $\boldsymbol{q}_{2}$ integration is done by the three-dimensional $\delta$ function, and the remaining phase-space integral is over $k_{1}, k_{2}, \theta_{q_{1} k_{1}}, \theta_{q_{1} k_{2}}$, and $\phi_{q_{1} ; k_{1} k_{2}}$, where $\theta_{q_{1} k_{1}}\left(\theta_{q_{1} k_{2}}\right)$ is the polar angle between $\boldsymbol{q}_{1}$ and $\boldsymbol{k}_{1}\left(\boldsymbol{k}_{2}\right)$ and $\phi_{q_{1} ; k_{1} k_{2}}$ is the azimuthal angle between the $\boldsymbol{q}_{1}-\boldsymbol{k}_{1}$ and $\boldsymbol{q}_{1}-\boldsymbol{k}_{2}$ planes. We introduce a dummy variable, $\int d \omega \delta\left(\omega-k_{1}+v k_{1} \cos \theta_{q_{1} k_{1}}\right)=$ 1 [8] and integrate over the polar angles using the remaining $\delta$ functions. From $Q_{2}^{2}=\left(Q_{1}-L\right)^{2}$ with the momentum transfer $L$, we obtain $Q_{1} \cdot L=\frac{L^{2}}{2}$. Then $k_{1}-k_{2}=$ $v k_{1} \cos \theta_{q_{1} k_{1}}-v k_{2} \cos \theta_{q_{1} k_{2}}-\frac{L^{2}}{2 q_{10}}$ and the energy conservation yields $\delta\left(k_{2}-\omega-v k_{2} \cos \theta_{q_{1} k_{2}}-\frac{L^{2}}{2 q_{10}}\right)$, where the last term $-\frac{L^{2}}{2 q_{10}}$ is negligible in comparison with the other terms of order $T$. Thus, with $\boldsymbol{k}_{1} \cdot \boldsymbol{k}_{2}=k_{1} k_{2}\left(\cos \theta_{q_{1} k_{1}} \cos \theta_{q_{1} k_{2}}+\right.$ $\left.\sin \theta_{q_{1} k_{1}} \sin \theta_{q_{1} k_{2}} \cos \phi_{q_{1} ; k_{1} k_{2}}\right)$, the energy loss is

$$
\begin{aligned}
-\frac{d E}{d x}= & \frac{1}{16 E(2 \pi)^{4} v^{3}} \int d \omega \int_{\frac{\omega}{1+v}}^{\frac{\omega}{1-v}} d k_{1} \int_{\frac{\omega}{1+v}}^{\frac{\omega}{1-v}} d k_{2} \\
& \times \int d \phi_{q_{1} ; k_{1} k_{2}} n\left(k_{1}\right)\left[1+n\left(k_{2}\right)\right] \\
& \times\left.\frac{\left(k_{2}-k_{1}\right)}{q_{20}}\left|\mathcal{M}_{\mathrm{el}}\right|^{2}\right|_{\cos \theta_{q_{1} k_{1}}=\frac{k_{1}-\omega}{k_{1} v}, \cos \theta_{q_{1} k_{2}}=\frac{k_{2}-\omega}{k_{2} v}},
\end{aligned}
$$




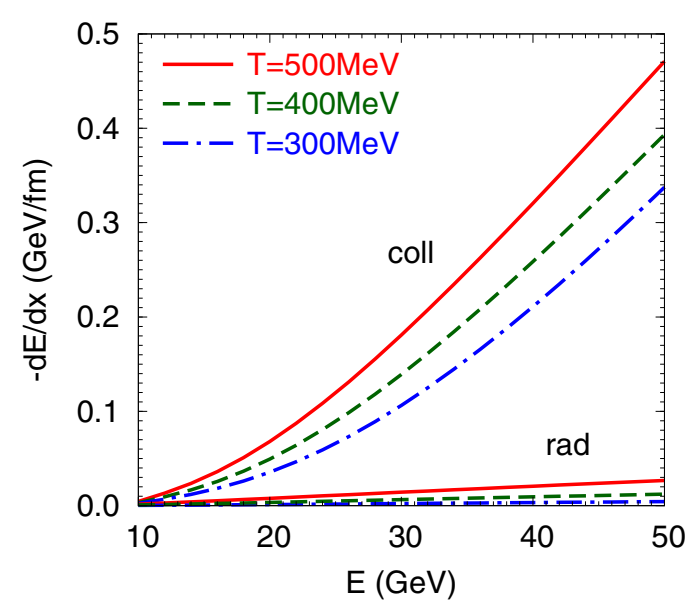

FIG. 2. The collisional and radiative energy loss of the $\Upsilon(1 S)$ in a plasma with temperature $T$, as a function of its initial energy. $\alpha_{s}=0.3$ is used.

which can be performed numerically by using the binding energy computed in lattice QCD [19].

The effective vertex of Eq. (1) is based on the dipole interaction of color charge with gluon which is valid when the quarkonium size is smaller than the inverse energy transfer of the gluon. Hence, we consider the quarkonium energy loss at a kinematic regime where the temperature and binding energy scales are smaller than $\frac{1}{a_{0}} \approx 1-1.5 \mathrm{GeV}$. In principle, there can be energy loss of the virtual heavy quarks in Fig. 1 [11] and energy loss coming from the color octet state prior to quarkonium formation. Assuming that the lifetime of the virtual heavy quarks (in the large- $N_{c}$ limit) and the color octet state is proportional to the formation time of the quarkonium which is related to the inverse of the binding energy, the square of the momentum transfer of the virtual heavy quarks and the initial color octet state is roughly of order $\frac{\kappa_{H Q}}{E_{b}} \sim \frac{g^{4} T^{3}}{E_{b}}$. In the regime $\frac{1}{r} \sim m g^{2}>T, E_{b} \sim m g^{4}>g^{2} T$, and the momentum transfer is smaller than the $g T$ scale. Since the momentum scale of the heavy quarks is at least of order $T$, we ignore the energy loss by the initial color octet state as well as the virtual heavy quark diffusion in this work.

Figure 2 shows our numerical results of the $\Upsilon(1 S)$ energy loss with $m=4.8 \mathrm{GeV}, m_{\Upsilon}=9.46 \mathrm{GeV}$, and $\alpha_{s}=0.3$. The collisional energy loss of the $\Upsilon(1 S)$ increases with its energy and the plasma temperature, and it is less than about half of the bottom quark energy loss $[2,7,10]$.

\section{RADIATIVE ENERGY LOSS}

Quarkonia undergoing quarkonium-gluon elastic scattering induce gluon radiation, and the amount of the emitted gluon energy is the radiative energy loss. In terms of the elastic scattering defined on the left-hand side of Fig. 1, the lowestorder contribution to the energy loss is from the processes in Fig. 3. Gluon emission from heavy quark lines is ignored in (a)

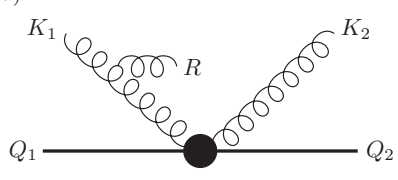

(b)

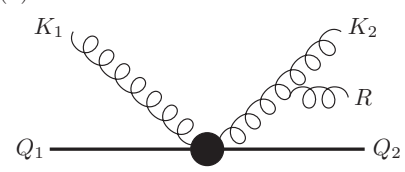

FIG. 3. Gluon-bremsstrahlung associated with quarkoniumgluon elastic scattering (Fig. 1).

the following light-cone coordinates [20]:

$$
\begin{aligned}
K_{1} & =\left[\sqrt{s}-\frac{m_{\Upsilon}^{2}}{\sqrt{s}}, 0, \mathbf{0}\right], \\
R & =\left[z\left(\sqrt{s}-\frac{m_{\Upsilon}^{2}}{\sqrt{s}}\right), \frac{r_{T}^{2}}{z\left(\sqrt{s}-\frac{m_{\Upsilon}^{2}}{\sqrt{s}}\right)}, \boldsymbol{r}_{T}\right], \\
Q_{1} & =\left[\frac{m_{\Upsilon}^{2}}{\sqrt{s}}, \sqrt{s}, \mathbf{0}\right], \quad L=\left[l^{+}, l^{-}, \boldsymbol{l}_{T}\right],
\end{aligned}
$$

where $z$ is the momentum fraction of the emitted gluon relative to the maximum available, and the components of the momentum transfer $L$ are determined by the on-shell conditions, $K_{2}^{2}=\left(K_{1}+L-R\right)^{2}=0$ and $Q_{2}^{2}=\left(Q_{1}-L\right)^{2}=m_{\Upsilon}^{2}$. In the gauge $A^{+}=0$, the polarization of the radiated gluon is specified by $\epsilon \cdot R=0$ and $\epsilon^{+}=0$,

$$
\epsilon=\left[0, \frac{2 \boldsymbol{\epsilon}_{T} \cdot \boldsymbol{r}_{T}}{z\left(\sqrt{s}-\frac{m_{\Upsilon}^{2}}{\sqrt{s}}\right)}, \boldsymbol{\epsilon}_{T}\right] .
$$

For soft gluon emission, the radiation processes are factorized into elastic scattering and gluon emission whose amplitude is the emitted gluon field multiplied by an additional gluon propagator. In the limit $r_{T} \gg l_{T}$, the leading contribution is as follows:

$$
\mathcal{M}_{\mathrm{rad}}=2 g C z \frac{\boldsymbol{\epsilon}_{T} \cdot \boldsymbol{r}_{T}}{\boldsymbol{r}_{T}^{2}} \mathcal{M}_{\mathrm{el}}
$$

where $C$ is the color factor associated with Fig. 3 divided by the factor in the absence of radiation $\left(C^{2}=3\right)$. The energy of the emitted gluon is small compared with that of the parent gluon for soft radiation, but it is still assumed to be larger than its transverse momentum.

The radiative energy loss associated with quarkoniumgluon elastic scattering can be determined by the emitted gluon spectrum,

$$
\begin{aligned}
\int d n_{g} & =\int \frac{d^{4} R}{(2 \pi)^{4}} 2 \pi \delta\left(R^{2}\right)\left|\frac{\mathcal{M}_{\mathrm{rad}}}{\mathcal{M}_{\mathrm{el}}}\right|^{2} \\
& =\int \frac{d z}{z} \int \frac{d^{2} \boldsymbol{r}_{T}}{(2 \pi)^{3}} 6 g^{2} \frac{z^{2}}{\boldsymbol{r}_{T}^{2}} .
\end{aligned}
$$

The mean energy loss is the average of the probability of emitting a gluon times the gluon energy,

$$
\delta E=\int d n_{g} r_{0}=\frac{g^{2}}{4 \pi^{2}}\left(\sqrt{s}-\frac{m_{\Upsilon}^{2}}{\sqrt{s}}\right) \ln \left[\frac{\sqrt{s}-\frac{m_{\Upsilon}^{2}}{\sqrt{s}}}{2 m_{D}}\right],
$$


where $r_{0}$ is the energy of the emitted gluon. In Eq. (12), the maximum transverse momentum of the gluon is given by $z k_{1}=z\left(\sqrt{s}-\frac{m_{r}^{2}}{\sqrt{s}}\right) / 2$, and the Debye screening mass $m_{D} \sim$ $g T$ has been chosen for the minimum: our results and the following discussion are not very sensitive to variation in the limits. For an estimate, we use $s \simeq m_{\Upsilon}^{2}+2 E k_{1}$ with a mean thermal energy $k_{1} \sim 3 T$. The average radiative energy loss per unit length is estimated by $-d E / d x \approx \delta E / \lambda$ with the wavelength $\lambda=1 /\left(\sigma_{\mathrm{el}} \rho\right)$, where $\sigma_{\mathrm{el}}$ is the cross section of quarkonium-gluon elastic scattering in Fig. 1 and $\rho=$ $16 \int \frac{d^{3} k}{(2 \pi)^{3}} n(k)=\frac{16 \zeta(3) T^{3}}{\pi^{2}}$ is the gluon density.

We present the $\Upsilon(1 S)$ radiative energy loss in Fig. 2, comparing with the collisional energy loss. The $\Upsilon(1 S)$ radiatively loses more energy in a hotter medium, but the effect is much smaller than the collisional energy loss, which grows more rapidly at high energy. In comparison with the radiative energy loss of a bottom quark [21], the energy loss of the $\Upsilon(1 S)$ is approximately an order of magnitude smaller at least.

\section{NUCLEAR MODIFICATION FACTOR}

In the previous two sections, we have computed the collisional and radiative energy loss of the $\Upsilon(1 S)$. Since the energy loss depends on the medium temperature, it changes with time as the plasma expands and cools down in heavy-ion collisions. In this section, we use the medium-induced energy loss to calculate the total energy loss during evolution, and then investigate the energy-loss effects on nuclear modification factors in the central rapidity region.

Quarkonium production has not been fully understood and the theoretical prediction involves large uncertainties even in the absence of a nuclear medium. For these reasons, we exploit quarkonium momentum spectra measured in $p p$ collisions and then estimate the energy loss effects in $A A$ collisions. The transverse momentum spectrum of the $\Upsilon(1 S)$ in $p p$ collisions can be parametrized as

$$
\frac{d \sigma_{\mathrm{pp}}}{d^{2} \boldsymbol{q}_{T}} \propto \frac{1}{\left[\left(\boldsymbol{q}_{T} / \Lambda\right)^{2}+1\right]^{\alpha}}
$$

$(\Lambda=6.05 \mathrm{GeV}$ and $\alpha=2.44)$ from the data in Ref. [15]. Using the momentum spectrum as an initial unquenched one, the energy-loss effects can be realized approximately by a shift of the momentum spectrum by the $\Upsilon(1 S)$ energy loss. For an expanding plasma undergoing a Bjorken expansion [22], $T(t)=T_{0}\left(\frac{t_{0}}{t}\right)^{1 / 3}$, the total energy loss during the evolution is $\Delta E=-\int_{t_{0}}^{t_{f}} d t v \frac{d E}{d x}$, where $t_{0} \sim 0.3 \mathrm{fm} / c$ and $t_{f} \sim 7 \mathrm{fm} / c$ at the phase transition (as assumed in Ref. [23]). If the energy loss is small $\left(\frac{\Delta E}{E} \ll 1\right.$, see Fig. 4), the effects on normalized transverse momentum spectra can be approximated as [24]

$$
\frac{d \sigma_{\mathrm{AA}}(E)}{d^{2} \boldsymbol{q}_{T}}=\frac{d \sigma_{\mathrm{pp}}(E+\Delta E)}{d^{2} \boldsymbol{q}_{T}} .
$$

Then we estimate the nuclear modification factor by the energy-loss effects,

$$
R_{\mathrm{AA}}\left(q_{T}\right)=\frac{\frac{d \sigma_{\mathrm{AA}}(E)}{d^{2} \boldsymbol{q}_{T}}}{\frac{d \sigma_{\mathrm{pp}}(E)}{d^{2} \boldsymbol{q}_{T}}} .
$$

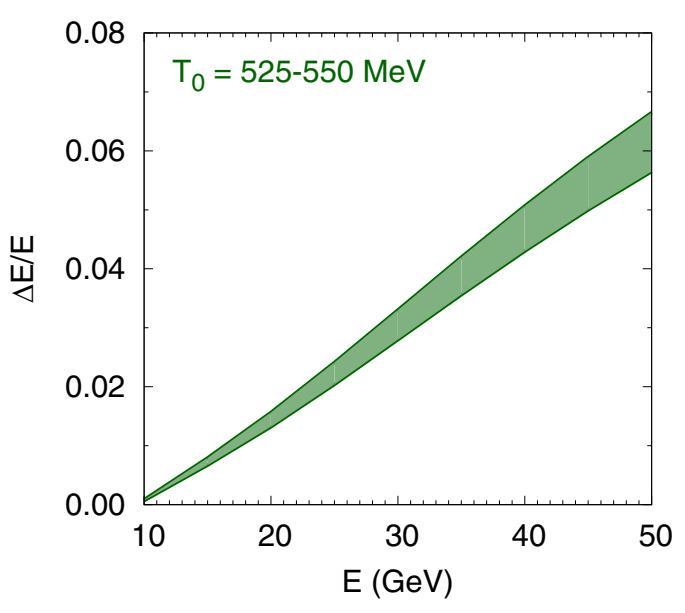

FIG. 4. The fractional energy loss $\frac{\Delta E}{E}$ of the $\Upsilon(1 S)$ for a Bjorken expansion with the initial temperature $T_{0}=525-550 \mathrm{MeV}$, the higher temperature being the upper boundary.

In Ref. [23] we have computed the nuclear modification factor of the $\Upsilon(1 S)$ by dissociation and regeneration in $\mathrm{PbPb}$ collisions at $\sqrt{s_{N N}}=2.76,5.02 \mathrm{TeV}$ and found that the numerical results depend on initial conditions with significant uncertainties at an early stage when quarkonia formation is in progress. The quarkonium energy loss can also affect the initial spectrum at the initial time QGP is formed (as well as during a Bjorken expansion): the energy-loss effects at the early stage might be important. Using the same initial conditions as Ref. [23] without the energy loss at the beginning, Fig. 5 shows the $\Upsilon(1 S) R_{\mathrm{AA}}$ factor determined by its energy loss during time evolution. Because the collisional energy loss increases with momentum as seen in Fig. 2, the $\Upsilon(1 S)$ is more suppressed at larger momentum. For higher initial temperature, we obtain stronger suppression.

The hot-medium effects can be obtained by including both quarkonium energy loss and dissociation-regeneration. In an effective-field theory framework, dissociation and

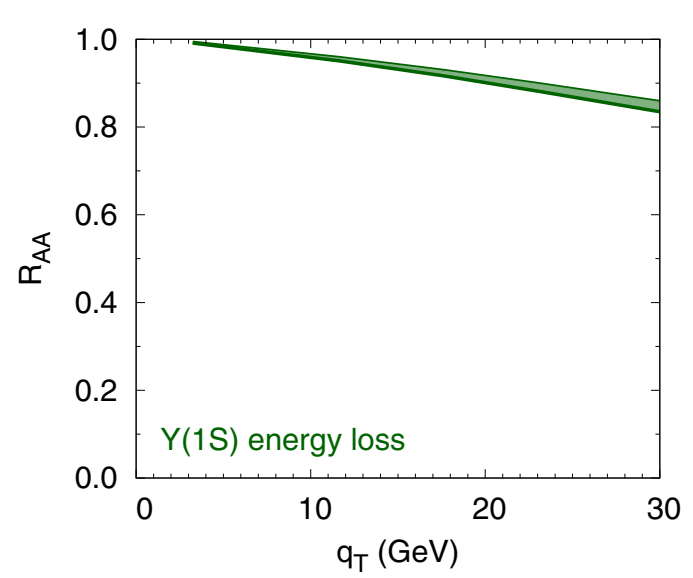

FIG. 5. The energy-loss effects on the $\Upsilon(1 S) R_{\mathrm{AA}}$ factor in an expanding plasma with the initial temperature $T_{0}=525-550$ $\mathrm{MeV}$ (as assumed in Ref. [23]) for heavy-ion collisions at $\sqrt{s_{N N}}=$ 2.76, $5.02 \mathrm{TeV}$. 
recombination have been systematically studied in Refs. [25-28]. Noting that the enhancement by regeneration tends to grow with momentum in Ref. [23], the energy loss as in Fig. 5 can weaken the enhancement influence at high momentum to be more consistent with the data. As the regeneration effects are more pronounced with softer initial distributions of quarkonia and heavy quarks, the energy-loss effects become more significant when the $\Upsilon(1 S)$ spectrum is softer. Although the energy loss makes a little contribution compared with the almost momentum-independent suppression $R_{\mathrm{AA}} \approx 0.4$ measured at the LHC $[15,29]$, the $\Upsilon(1 S)$ energy loss can affect the high-momentum spectra up to $\approx 15 \%$ at $q_{T} \approx 30 \mathrm{GeV}$, and thus the diffusion and energy loss of heavy quarkonia, together with dissociation and regeneration, need to be taken into account to analyze the experimental data.

For feed-down, we can apply the calculation of quarkonium energy loss to the excited states of bottomonia as well. Using a Coulombic bound state, $\left|\nabla \psi_{2 S}(\boldsymbol{p})\right|^{2}=$ $2^{7} \pi a_{0}^{7} \boldsymbol{p}^{2}\left[\left(a_{0} \boldsymbol{p}\right)^{2}-\frac{1}{2}\right]^{2} /\left[\left(a_{0} \boldsymbol{p}\right)^{2}+\frac{1}{4}\right]^{8}$, with a quarter of the binding energy of the ground state, the energy loss of the $\Upsilon(2 S)$ is more than six times as large as the $\Upsilon(1 S)$ energy loss. Larger energy loss as well as thermal width of excited states might lead to sequential suppression of bottomonia.

\section{SUMMARY}

We have presented an estimate of quarkonium energy loss in hot QCD plasmas using an effective vertex between quarkonium and gluon. Based on our formalism derived from the Bethe-Salpeter amplitude, we have investigated the leading effects on the transverse momentum spectra of $\Upsilon(1 S)$. The collisional energy loss is obtained by convoluting the interaction rate of quarkonium-gluon elastic scattering and the energy transfer. The radiative energy loss is realized through gluon-bremsstrahlung associated with the elastic scattering and is estimated by a convolution of the probability of emitting a gluon and its energy. Our numerical analysis indicates that the collisional energy loss is dominant over the radiative one in the energy regime $E<m_{\Upsilon}^{2} / T$ and increases with the quarkonium energy and the plasma temperature. In comparison with the bottom quark energy loss, the energy loss of the $\Upsilon(1 S)$ is smaller by a factor of two at least.

The energy loss of heavy quarkonia affects their transverse momentum spectra and is reflected on the nuclear modification factors, resulting in quarkonia suppression. Adopting a simple shift of the momentum spectra by the energy loss for a Bjorken expansion, we have estimated the energy-loss effects on the $\Upsilon(1 S) R_{\mathrm{AA}}$ factor. The energy loss is found to provide a small effect on the $\Upsilon(1 S)$ suppression compared with quarkonium dissociation, but it is necessary to consider the hot-medium effects and to analyze the experimental data in heavy-ion collisions. Furthermore, the energy loss of heavy quarkonia might be partially responsible for sequential suppression even in small systems. As the medium-induced energy loss is sensitive to many factors including path length and geometrical effects in nuclear collisions, the mean energy loss is not sufficient and more systematic studies need to be undertaken.

\section{ACKNOWLEDGMENTS}

We would like to thank Yongsun Kim for useful discussions. This work is supported by the National Research Foundation of Korea (NRF) grant funded by the Korea government (MSIT) (No. 2018R1C1B6008119).
[1] E. Braaten and M. H. Thoma, Phys. Rev. D 44, 1298 (1991).

[2] E. Braaten and M. H. Thoma, Phys. Rev. D 44, R2625 (1991).

[3] M. Gyulassy and X.-N. Wang, Nucl. Phys. B 420, 583 (1994).

[4] X.-N. Wang, M. Gyulassy, and M. Plumer, Phys. Rev. D 51, 3436 (1995).

[5] R. Baier, Yu. L. Dokshitzer, A. H. Mueller, S. Peigné, and D. Schiff, Nucl. Phys. B 483, 291 (1997).

[6] R. Baier, Yu. L. Dokshitzer, A. H. Mueller, S. Peigné, and D. Schiff, Nucl. Phys. B 484, 265 (1997).

[7] M. G. Mustafa, D. Pal, D. K. Kumar, and M. Thoma, Phys. Lett. B 428, 234 (1998); 438, 450 (1998).

[8] G. D. Moore and D. Teaney, Phys. Rev. C 71, 064904 (2005).

[9] M. G. Mustafa, Phys. Rev. C 72, 014905 (2005).

[10] M. Djordjevic, Phys. Rev. C 74, 064907 (2006).

[11] X. Yao and B. Müller, Phys. Rev. D 100, 014008 (2019).

[12] J. Hong and S. H. Lee, Phys. Rev. C 99, 034905 (2019).

[13] G. Bhanot and M. E. Peskin, Nucl. Phys. B 156, 391 (1979).

[14] N. Brambilla, M. A. Escobedo, J. Ghiglieri, and A. Vairo, J. High Energy Phys. 05 (2013) 130.

[15] A. M. Sirunyan et al. (CMS Collaboration), Phys. Lett. B 790, 270 (2019).
[16] A. Mocsy, P. Petreczky, and M. Strickland, Int. J. Mod. Phys. A 28, 1340012 (2013).

[17] T. Song and S. H. Lee, Phys. Rev. D 72, 034002 (2005).

[18] K. Dusling, D. Teaney, C. Young, J. Erdmenger, M. Kaminski, and F. Rust, J. High Energy Phys. 10 (2008) 098.

[19] A. Mocsy and P. Petreczky, Phys. Rev. Lett. 99, 211602 (2007).

[20] J. F. Gunion and G. Bertsch, Phys. Rev. D 25, 746 (1982).

[21] M. Djordjevic and M. Gyulassy, Nucl. Phys. A 733, 265 (2004).

[22] J. D. Bjorken, Phys. Rev. D 27, 140 (1983).

[23] J. Hong and S. H. Lee, Phys. Lett. B 801, 135147 (2020).

[24] R. Baier, Y. L. Dokshitzer, A. H. Mueller, and D. Schiff, J. High Energy Phys. 09 (2001) 033.

[25] X. Yao and B. Müller, Phys. Rev. C 97, 014908 (2018); 97, 049903(E) (2018).

[26] X. Yao and T. Mehen, Phys. Rev. D 99, 096028 (2019).

[27] X. Yao, W. Ke, Y. Xu, S. A. Bass, and B. Müller, J. High Energy Phys. 01 (2021) 046.

[28] X. Yao and T. Mehen, J. High Energy Phys. 02 (2021) 062.

[29] V. Khachatryan et al. (CMS Collaboration), Phys. Lett. B 770, 357 (2017). 